\title{
ON LOCALLY CONFORMAL KÄHLER SPACE FORMS
}

\section{KOJI MATSUMOTO}

Department of Mathematics

Faculty of Education

Yamagata University

Yamagata, 990, Japan

(Received May 29, 1984)

ABSTRACT. An m-dimensional locally conformal KHhler manifold (1.c.K-manifold) is characterized as a Hermitian manifold admitting a global closed 1 -form $\alpha_{\lambda}$ (called the Lee form) whose structure $\left(F_{\mu}{ }^{\lambda}, g_{\mu \lambda}\right)$ satisfies

$$
\nabla_{\nu} F_{\mu \lambda}=-\beta_{\mu} g_{\nu \lambda}+\beta_{\lambda} g_{\nu \mu}-\alpha_{\mu} F_{\nu \lambda}+\alpha_{\lambda} F_{\nu \mu}
$$

where $\nabla_{\lambda}$ denotes the covariant differentiation with respect to the Hermitian metric $g_{\mu \lambda}, B_{\lambda}=-F_{\lambda}{ }^{\varepsilon} \alpha_{\varepsilon}, F_{\mu \lambda}=F_{\mu}{ }^{\varepsilon} g_{\varepsilon \lambda}$ and the indices $\nu, \mu, \ldots, \lambda$ run over the range $1,2, \ldots, m$. For 1.c.K-manifolds, I.Vaisman [4] gave a typical example and T.Kashiwada ([1], [2],[3]) gave a lot of interesting properties about such manifolds.

In this paper, we shall study certain properties of $1 . \mathrm{c} . \mathrm{K}$-space forms. In $\$ 2$, we shall mainly get the necessary and sufficient condition that an 1.c.K-space form is an Einstein one and the Riemannian curvature tensor with respect to $g_{\mu \lambda}$ will be expressed without the tensor field $P_{\mu \lambda}$. In $\S 3$, we shall get the necessary and sufficient condition that the length of the Lee form is constant and the sufficient condition that a compact 1.c.K-space form becomes a complex space form. In the last $\$ 4$, we shall prove that there does not exist a non-trivial recurrent 1.c.K-space form.

KEY WORDS \& PHRASES: L.c.K-manifolds, Lee form, l.c.K-space forms, hybrid, recurrent l.c. K-space form.

1980 MATHEMATICS SUBJECT CLASSIFICATION CODE. $53 B 25$

\section{INTRODUCTION.}

This paper is directed to specialist readers with background in the area and appreciative of its relation of this area of study.

Let $M\left(F_{\mu}^{\lambda}, g_{\mu \lambda}, \alpha_{\lambda}\right)$ be an 1.c.K-manifold. Then, by the definition, at any point of $M$ there exists a neighborhood in which a conformal metric $g^{*}=e^{-2 \rho} g$ is a kHhler one, i.e.,

$$
\nabla_{\nu}^{*}\left(e^{-2 \rho_{F}}{ }_{\mu \lambda}\right)=0, \quad d \rho=\alpha,
$$

where $\nabla_{\lambda}^{*}$ denotes the covariant differentiation with respect to $g^{*}$. Then we have 


$$
\nabla_{\nu} F_{\mu \lambda}=-\alpha_{\mu} F_{\nu \lambda}+\alpha^{\varepsilon_{\varepsilon \lambda}} F_{\nu \mu}+\alpha_{\lambda} F_{\nu \mu}+\alpha^{\varepsilon F_{\mu \varepsilon} g_{\nu \lambda}} .
$$

The following proposition was proved by T.Kashiwada [1]

PROPOSITION 1.1. A Hermitian manifold $M\left(F_{\mu}{ }_{\mu}, g_{\mu \lambda}\right)$ is an 1.c.K-manifold if and only if there exists a global closed 1-form $\alpha_{\lambda}$ satisfying (1.1).

In an 1.c.K-manifold $M$, we define a tensor field $P_{\mu \lambda}$ as follows;

$$
P_{\mu \lambda}=-\nabla_{\mu} \alpha_{\lambda}-\alpha_{\mu \lambda} \alpha_{\lambda}+\frac{1}{2}\|\alpha\|^{2} g_{\mu \lambda},
$$

where $\|\alpha\|$ denotes the length of the Lee form $\alpha_{\lambda}$ with respect to $g_{\mu \lambda}$.

In an $m$-dimensional 1.c.K-manifold $M$, we know the following formula;

$$
R_{\mu \varepsilon}{ }^{F_{\lambda}}{ }^{\varepsilon}+R_{\lambda \varepsilon^{F_{\mu}}}{ }^{\varepsilon}-(m-2)\left(P_{\mu \varepsilon}{ }^{F_{\lambda}}{ }^{\varepsilon}+P_{\lambda \varepsilon^{F_{\mu}}}{ }^{\varepsilon}\right)=0,
$$

where $R_{\mu \lambda}$ denotes the Ricci tensor with respect to $g_{\mu \lambda}[1]$. Thus we have

PROPOSITION 1.2. In an $m$-dimensional $(m \neq 2)$ 1.c.K-manifold $M$, the tensor field $P_{\mu \lambda}$ is hybrid, i.e.,

$$
P_{\mu \varepsilon}{ }^{F}{ }^{\varepsilon}+P_{\lambda \varepsilon^{F}}^{F_{\mu}^{\varepsilon}}=0,
$$

if and only if the Ricci tensor $R_{\mu \lambda}$ is hybrid.

From now on in this paper, we assume that the tensor field $P_{\mu \lambda}$ is hybrid.

REMARK. In an $m$-dimensional $(m \neq 2)$ Einstein $1 . c . K$-manifold, the tensor field $P_{\mu \lambda}$ is hybrid, identically.

An 1.c.K-manifold $M$ is called an 1.c.K-space form if the holomorphic sectional curvature of the section $\{X, F X\}$ at each point of $M$ has the constant value. Let $M(H)$ be an 1.c.K-space form with constant holomorphic sectional curvature $H$. Then the Riemannian curvature tensor $R_{\omega \nu \mu \lambda}$ with respect to $g_{\mu \lambda}$ can be written as

$$
\begin{aligned}
& { }^{4 R} R_{\omega \nu \lambda}=H\left(g_{\omega \lambda} g_{\nu \mu}-g_{\omega \mu} g_{\nu \lambda}+F_{\omega \lambda} F_{\nu \mu}-F_{\omega \mu} F_{\nu \lambda}-2 F_{\omega \nu} F_{\mu \lambda}\right)+3\left(P_{\omega \lambda} g_{\nu \mu}-P_{\omega \mu} g_{\nu \lambda}\right. \\
& \left.+g_{\omega \lambda} P_{\nu \mu}-g_{\omega \mu} P_{\nu \lambda}\right)-\left\{\tilde{P}_{\omega \lambda} F_{\nu \mu}-\tilde{P}_{\omega \mu} F_{\nu \lambda}+F_{\omega \lambda} \tilde{P}_{\nu \mu}-F_{\omega \mu} \tilde{P}_{\nu \lambda}-2\left(\tilde{P}_{\omega \nu} F_{\mu \lambda}\right.\right. \\
& \left.\left.+F_{\omega \nu} \tilde{P}_{\mu \lambda}\right)\right\}
\end{aligned}
$$

where $\tilde{P}_{\mu \lambda}=P_{\mu} \varepsilon_{F \lambda}[1]$.

2. L.C.K-SPACE FORMS.

In this section, we shall consider the necessary and sufficient condition that an 1.c.K-space form becomes an Einstein one. Next, we shall get an expression of the Riemannian curvature $R_{\omega \nu \mu \lambda}$ that does not include the tensor field $P_{\mu \lambda}$.

Let $M(H)$ ba an m-dimensional 1.c.K-space form with constant holomorphic sectional curvature $H$. Then we have (1.5). Transvecting (1.5) with $g^{\omega \lambda}$, we have from the straightfoward calculation

$$
4 R_{\mu \lambda}=\left\{(m+2) H+3 P g_{\mu \lambda}+3(m-4) P_{\mu \lambda},\right.
$$

where $P=P_{\mu \lambda} g^{\mu \lambda}$ and it can be written as

Thus we have

$$
P=-\nabla_{\varepsilon} \alpha^{\varepsilon}+\frac{1}{2}(m-2)\|\alpha\|^{2} .
$$

PROPOSITION 2.1. A 4-dimensional 1.c.K-space form $M(H)$ which the tensor field $P_{\mu \lambda}$ is hybrid is an Einstein one and then the scalar field $P$ is constant. We have from (2.2) and the Green's theorem [5] 
PROPOSITION 2.2. A compact m-dimensional 1.c.K-space form $M(H)$ which the tensc $r$ field $P_{\mu \lambda}$ is hybrid has a non-negative $P$.

Next, we shall prove the following;

THEOREM 2.3. An $m$-dimensional $(m \neq 4)$ 1.c.K-space form $M(H)$ which the tensor field $P_{\mu \lambda}$ is hybrid is an Einstein one if and only if the tensor field $P_{\mu \lambda}$ is proportional to $g_{\mu \lambda}$.

PROOF. If the tensor field $P_{\mu \lambda}$ is proportional to $g_{\mu \lambda}$, then the tensor field $P_{\mu \lambda}$ can be written as

$$
P_{\mu \lambda}=\frac{P}{m} g_{\mu \lambda}
$$

Thus we have from (2.1) and (2.3)

$$
R_{\mu \lambda}=\left\{(m+2) H+\frac{6(m-2)}{m} P\right\}_{\mu \lambda} \text {. }
$$

The inverse is trivial, so we omit its proof.

COROLLARY 2.4. An $m$-dimensional $(m \neq 4)$ Einstein 1.c.K-space form $M(H)$ which the tensor field $P_{\mu \lambda}$ is hybrid is a complex space form if $P=0$.

Transvecting (2.1) with $g^{\mu \lambda}$, we have

$$
4 R=m(m+2) H+6(m-2) P,
$$

where $R$ denotes the scalar curvature with respect to $g_{\mu \lambda}$. By virtue of (2.1) and (2.4), we can easily see that

$$
\begin{aligned}
& 3 P_{\mu \lambda}=\frac{4}{m-4}{ }_{\mu \lambda}-\frac{(m-4)(m+2) H+4 R}{2(m-2)(m-4)} g_{\mu \lambda}, \\
& \tilde{P}_{\mu \lambda}=\frac{4}{3(m-4)} \tilde{R}_{\mu \lambda}-\frac{(m-4)(m+2) H+4 R_{F}}{6(m-2)(m-4)} F_{\mu \lambda}
\end{aligned}
$$

where $\tilde{R}_{\mu \lambda}=R_{\mu}^{\varepsilon_{F}}{ }_{\varepsilon \lambda}$. Substituting (2.5) and (2.6) into (1.5), we obtain

$$
\begin{aligned}
& R_{\omega \nu \mu \lambda}=-\frac{(m-4) H+R}{(m-2)(m-4)}\left(g_{\omega \lambda} g_{\nu \mu}-g_{\omega \mu} g_{\nu \lambda}\right)+\frac{(m-4)(m-1) H+R}{3(m-2)(m-4)}\left(F_{\omega \lambda} F_{\nu \mu}\right. \\
& \left.-F_{\omega \mu} F_{\nu \lambda}-2 F_{\omega \nu} F_{\mu \lambda}\right)+\frac{1}{(m-4)}\left(R_{\omega \lambda} g_{\nu \mu}-R_{\omega \mu} g_{\nu \lambda}+g_{\omega \lambda} R_{\nu \mu}-g_{\omega \mu \mu} R_{\nu \lambda}\right) \\
& +\frac{1}{3(m-4)}\left\{\tilde{R}_{\omega \lambda} F_{\nu \mu}-\tilde{R}_{\omega \mu \nu \lambda} F_{\nu \lambda}+F_{\omega \lambda} \tilde{R}_{\nu \mu}-F_{\omega \mu} \tilde{R}_{\nu \lambda}-2\left(\tilde{R}_{\omega \nu} F_{\mu \lambda}+F_{\omega \nu} \tilde{R}_{\mu \lambda}\right)\right\} .
\end{aligned}
$$

Thus we have

PROPOSITION 2.5. In an $m$-dimensional $(m \neq 2,4)$ 1.c.K-space form $M(H)$ which the tensor field $P_{\mu \lambda}$ is hybrid, the Riemannian curvature tensor $R_{\omega \nu \mu \lambda}$ can be written as (2.7) without $P_{\mu \lambda}$.

3. COMPACT L.C.K-SPACE FORMS.

In this section, we shall mainly deal with compact 1.c.K-space form.

Let $M(H)$ be an $m$-dimensional 1.c.K-space form with constant holomorphic sectional curvature $H$. If we assume that the scalar curvature $R$ is constant, then by virtue of (2.4) all of the scalar fields $R, H$ and $P$ are constant. Under this assumption, differentiating (2.1) covariantly, we get

$$
4 \nabla_{\omega} R_{\nu \mu}=3(m-4) \nabla_{\omega} P_{\nu \mu}
$$

Substituting (1.2) into the above equation, we have

$$
4 \nabla_{\omega} R_{\nu \mu}=3(m-4)\left\{-\nabla_{\omega} \nabla_{\nu} \alpha_{\mu}-\left(\nabla_{\omega} \alpha_{\nu}\right) \alpha_{\mu}-\alpha_{\nu} \nabla_{\omega} \alpha_{\mu}+\frac{1}{2}\left(\nabla_{\omega}\|\alpha\|^{2}\right) g_{\nu \mu}\right\} .
$$

By virtue of the Ricci identity [5] and the assumption $\nabla_{\mu} \alpha_{\lambda}=\nabla_{\lambda} \alpha_{\mu}$, the equation (3.2) implies 


$$
\begin{aligned}
& 4\left(\nabla_{\omega} R_{\nu \mu}-\nabla_{\nu} R_{\omega \mu}\right)=3(m-4)\left\{R_{\omega \nu \mu} \varepsilon_{\varepsilon}+\alpha_{\omega}\left(\nabla_{\nu} \alpha_{\mu}\right)-\alpha_{\nu}\left(\nabla_{\omega} \alpha_{\mu}\right)\right. \\
& \left.+\frac{1}{2}\left(\nabla_{\omega}\|\alpha\|^{2} g_{\nu \mu}-\nabla_{\nu}\|\alpha\|^{2} g_{\omega \mu}\right)\right\} .
\end{aligned}
$$

Transvecting the above equation with $g^{\nu \mu}$ and taking account of the formula $2 \nabla_{\varepsilon} R_{\lambda}^{\varepsilon}=$ $\nabla_{\lambda} R[5]$, we obtain

$$
R_{\omega}^{\varepsilon} \alpha_{\varepsilon}+\left(\nabla_{\varepsilon} \alpha^{\varepsilon}\right) \alpha_{\omega}+\frac{1}{2}(m-2) \nabla_{\omega}\|\alpha\|^{2}=0 .
$$

Substituting (2.1) into (3.3), we obtain

$$
\left\{(m+2) H+3\|\alpha\|^{2}+\nabla_{\varepsilon} \alpha^{\varepsilon}\right\} \alpha_{\omega}+\frac{m-4}{2} \nabla_{\omega}\|\alpha\|^{2}=0 .
$$

Thus we have

THEOREM 3.1. In an $m$-dimensional $(m \neq 2,4)$ 1.c.K-space form $M(H)$ which the tensor field $P_{\mu \lambda}$ is hybrid and the scalar curvature $R$ is constant, the length $\|\alpha\|$ of the Lee form $\alpha_{\lambda}$ is non-zero constant if and only if

$$
(m+2) H+3\|\alpha\|^{2}+\nabla_{\varepsilon} \alpha^{\varepsilon}=0 \text {. }
$$

By virtue of (3.5) and the Green's theorem, we have

COROLLARY 3.2. In a compact $m$-dimensional $(m \neq 2,4)$ 1.c.K-space form $M(H)$ which the tensor field $P_{\mu \lambda}$ is hybrid and the scalar curvature $R$ is constant, if the length $\|\alpha\|$ of the Lee form $\alpha_{\lambda}$ is non-zero constant, then there exists the following relation between the holomorphic sectional curvature $H$ and the length $\|\alpha\|$ of the Lee form $\alpha_{\lambda}$;

$$
(m+2) H+3\|\alpha\|^{2}=0 .
$$

COROLLARY 3.3. There does not exist a compact m-dimensional $(m \neq 2,4) 1 . \mathrm{c} . \mathrm{K}-$ space form $M(H)$ which the tensor field $P_{\mu \lambda}$ is hybrid and the holomorphic sectional curvature $H$ is positive if the length $\|\alpha\|$ of the Lee form $\alpha_{\lambda}$ and the scalar curvature $R$ are constant. Especially, if $H=0$, then the manifold $M$ must be locally Euclidean, that is, the Riemannian curvature tensor $R_{\omega \nu \mu \lambda}$ is identically zero.

The following proposition was proved by T.Kashiwada [1];

PROPOSITION 3.4. In a compact m-dimensiona1 $(m \neq 2) 1$. . K-manifold $M$, if

$$
\tilde{H}_{\varepsilon}^{\varepsilon}-R \geqslant 0
$$

holds good, then the manifold $M$ is a KHhler manifold, where $\tilde{H}_{\mu \lambda}=\frac{1}{2} R_{\mu}{ }^{\varepsilon} \delta \gamma^{F}{ }^{\delta \gamma_{F}} \varepsilon_{\varepsilon}$. The inequality $\geqslant$ in this case is naturally reduced to $=$.

Now, let $M(H)$ be a compact m-dimensional $(m \neq 2,4) 1$.c.K-space form. Then transvecting (2.5) with $F^{\omega \nu_{F}} \mu \lambda$, we get

$$
\frac{1}{2} R_{\omega \nu \mu \lambda} F^{\omega \nu_{F} \mu \lambda}=\frac{-m(m+2) H+R}{3} \text {. }
$$

By virtue of (2.4) and (3.8), we obtain

$$
H_{\varepsilon}^{\varepsilon}-R=\frac{m(m+2) H-4 R}{3} \text {. }
$$

Thus we have from PROPOSITION 3.4 and (3.9)

THEOREM 3.5. In a compact $m$-dimensional $(m \neq 2,4)$ 1.c.K-space form $M(H)$ which the tensor field $P_{\mu \lambda}$ is hybrid, if the inequlity $m(m+2) H \geqslant 4 R$ holds good, then the manifold $M$ is a complex space form.

4. RECURRENT L.C.K-SPACE FORMS.

A Riemannian manifold $M$ is said to be recurrent if the Riemannian curvature tensor 
$R_{\omega \nu \mu \lambda} \begin{gathered}\text { satisfies } \\ \nabla_{\kappa} R_{\omega \nu \mu \lambda}\end{gathered}=\theta_{\kappa} R_{\omega \nu \mu \lambda}$

for a certain non-zero vector field $\theta_{K}$. For a recurrent Riemannian manifold, it is trivial that

$$
\nabla_{\nu} R_{\mu \lambda}=\theta_{\nu} R_{\mu \lambda}, \quad \nabla_{\lambda} R=\theta_{\lambda} R .
$$

Now, let $M(H)$ be an $m$-dimensional $(m \neq 2,4)$ recurrent $1 . c . K$-space form which the tensor field $P_{\mu \lambda}$ is hybrid. Then we have (2.7) and (4.1). Differentiating (2.7) covariantly and taking account of (4.1) and (4.2), we have

$$
\begin{aligned}
& \frac{H}{m-2} \theta_{\kappa}\left(g_{\omega \lambda} g_{\nu \mu}-g_{\omega \mu} g_{\nu \lambda}\right)-\frac{(m-1) H}{3(m-2)} \theta_{\kappa}\left(F_{\omega \lambda} F_{\nu \mu}-F_{\omega \mu} F_{\nu \lambda}-2 F_{\omega \nu} F_{\mu \lambda}\right) \\
& +\frac{(m-4)(m-1) H+R}{3(m-2)(m-4)}\left\{\left(g_{\kappa \mu} F_{\nu \lambda}-g_{\kappa \lambda} F_{\nu \mu}+2 g_{K \nu} F_{\mu \lambda}\right) \beta_{\omega}+\left(g_{\kappa \lambda} F_{\omega \mu}-g_{K \mu} F_{\omega \lambda}\right.\right. \\
& \left.-2 g_{K \omega} F_{\mu \lambda}\right) B_{\nu}+\left(g_{K \nu} F_{\omega \lambda}-g_{K \omega} F_{\nu \lambda}+2 g_{K \lambda} F_{\omega \nu}\right) \beta_{\mu}+\left(g_{K \omega} F_{\nu \mu}-g_{K \nu} F_{\omega \mu}-2 g_{K \mu} F_{\omega \nu}\right) B_{\lambda} \\
& +\left(F_{\kappa \mu} F_{\nu \lambda}-F_{\kappa \lambda} F_{\nu \mu}+2 F_{\kappa \nu} F_{\mu \lambda}\right) \alpha_{\omega}+\left(F_{\kappa \lambda} F_{\omega \mu}-F_{\kappa \mu} F_{\omega \lambda}-2 F_{K \omega} F_{\mu \lambda}\right) \alpha_{\nu} \\
& \left.+\left(F_{\kappa \nu} F_{\omega \lambda}-F_{\kappa \omega} F_{\nu \lambda}+2 F_{\kappa \lambda} F_{\kappa \nu}\right) \alpha_{\mu}+\left(F_{\kappa \omega} F_{\nu \mu}-F_{\kappa \nu} F_{\omega \mu}-2 F_{\omega \nu} F_{\kappa \mu}\right) \alpha_{\lambda}\right\} \\
& -\frac{1}{3(m-4)}\left[\left\{R_{\omega}^{\varepsilon} g_{\kappa \mu} F_{\nu \lambda}-R_{\omega}^{\varepsilon} g_{\kappa \lambda} F_{\nu \mu}-R_{\nu}^{\varepsilon} g_{\kappa \mu} F_{\omega \lambda}+R_{\nu}^{\varepsilon} g_{\kappa \lambda} F_{\omega \mu}+2\left(R_{\omega}^{\varepsilon} g_{\kappa \nu} F_{\mu \lambda}\right.\right.\right.
\end{aligned}
$$

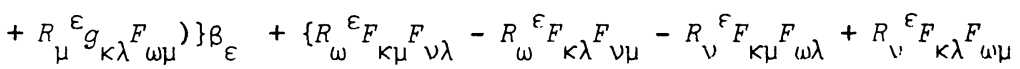

$$
\begin{aligned}
& \left.+2\left(R_{\omega} F_{K \nu} F_{\mu \lambda}+R_{\mu} F_{\kappa \lambda} F_{\omega \nu}\right)\right\}_{\varepsilon}+\left(g_{\kappa \mu} \tilde{R}_{\nu \lambda}-g_{\kappa \lambda} \tilde{R}_{\nu \mu}+2 g_{\kappa \nu} \tilde{R}_{\mu \lambda}\right) \beta_{\omega}+\left\{g_{\kappa \lambda} \tilde{R}_{\omega \mu}\right. \\
& \left.-g_{\kappa \mu} \tilde{R}_{\omega \lambda}-2\left(F_{\mu \lambda} R_{\omega \kappa}+g_{\kappa \omega} \tilde{R}_{\mu \lambda}\right)\right\} \beta_{\nu}+\left(g_{\kappa \nu} \tilde{R}_{\omega \lambda}-F_{\nu \lambda} R_{\omega K}+F_{\omega \lambda} R_{\kappa \nu}-g_{\kappa \omega} \tilde{R}_{\nu \lambda}\right. \\
& \left.+2 g_{\kappa \lambda} \tilde{R}_{\omega \nu}\right) B_{\mu}+\left\{F_{\nu \mu} R_{\omega K}-g_{K \nu} \tilde{R}_{\omega \mu}+g_{\kappa \omega} \tilde{R}_{\nu \mu}-F_{\omega \mu} R_{\kappa \nu}-2\left(g_{\omega \nu} \tilde{R}_{K \mu}+F_{\omega \nu} R_{K \mu}\right)\right\} B_{\lambda} \\
& +\left(F_{\kappa \mu} \tilde{R}_{\nu \lambda}-F_{\kappa \lambda} \tilde{R}_{\nu \mu}+2 F_{\kappa \nu} \tilde{R}_{\mu \lambda}\right) \alpha_{\omega}+\left\{F_{\kappa \lambda} \tilde{R}_{\omega \mu}-F_{\kappa \mu} \tilde{R}_{\omega \lambda}-2\left(F_{\mu \lambda} \tilde{R}_{\kappa \omega}+F_{\kappa \omega} \tilde{R}_{\mu \lambda}\right)\right\} \alpha_{\nu} \\
& +\left(F_{\kappa \nu} \tilde{R}_{\omega \lambda}-F_{\nu \lambda} \tilde{R}_{\kappa \omega}+F_{\omega \lambda} \tilde{R}_{\kappa \nu}-F_{\kappa \omega} \tilde{R}_{\nu \lambda}+2 F_{\kappa \lambda} \tilde{R}_{\omega \nu}\right) \alpha_{\mu}+\left\{F_{\nu \mu} \tilde{R}_{\kappa \omega}-F_{\kappa \nu} \tilde{R}\right. \\
& \left.\left.+F_{\kappa \omega} \tilde{R}_{\nu \mu}-F_{\omega \mu} \tilde{R}_{\kappa \nu}-2\left(F_{\kappa \mu} \tilde{R}_{\omega \nu}+F_{\omega \nu} \tilde{R}_{\kappa \mu}\right)\right\}_{\alpha_{\lambda}}\right]=0 .
\end{aligned}
$$

Transvecting (4.3) with $F^{(\mu \lambda \lambda}$, we get

$$
\begin{aligned}
& \frac{(m+2) H_{0}}{3} \theta_{K \nu \mu}=\frac{(m+2)\{(m-4)(m-1) H+R\}}{3(m-4)(m-2)}\left(g_{K \nu} \beta_{\mu}-g_{K \mu} \beta_{\nu}-F_{K \mu} \alpha_{\nu}+F_{K \nu} \alpha_{\mu}\right) \\
& \frac{1}{3(m-4)}\left[\left\{(m-1) R_{\nu} \varepsilon_{F_{K \mu}}-5 R_{\mu} \varepsilon_{F_{K \nu}}\right\} \alpha_{\varepsilon}+\left\{(m-1) R_{\nu} g_{K \mu}-5 R_{\mu} g_{K \nu}\right\} \beta_{\varepsilon}\right. \\
& +\left(R F_{K \mu}+5 R_{K \mu}\right) \alpha_{\nu}-\left\{R F_{K \nu}+(m-1) R_{K \nu}\right\} \alpha_{\mu}+\left(R g_{K \mu}+5 R_{K \mu}\right) \beta_{\nu} \\
& \left.-\left\{R g_{K \nu}+(m-1) R_{K \nu}\right\} \beta_{\mu}\right] .
\end{aligned}
$$

From this, we obtain

$$
H \theta_{K}=0 \text {. }
$$

Thus we have

THEOREM 4.1. An $m$-dimensional $(m \neq 2,4)$ recurrent 1.c.K-space form $M(H)$ which the tensor field $P_{\mu \lambda}$ is hybrid is trivial, that is, the manifold is locally symmetric or of zero holomorphic sectional curvature.

Let $M(H)$ be a 4-dimensional recurrent 1 .c.K-space form. Then, by virtue of PROPOSITION 2.1, the manifold is Einstein. Thus we have from (2.1) and (4.2)

$$
(2 H+P) \theta_{K}=0 \text {. }
$$


Thus we have

THEOREM 4.2. A 4-dimensional recurrent 1.c.K-space form $M(H)$ which the tensor field $P_{\mu \lambda}$ is hybrid is trivial or the manifold has a property $2 H+P=0$.

\section{REFERENCES}

1. VAISMAN, I. On Locally Conformal Almost Kähler Manifolds, Isurael J. Math., $\underline{24}$ (1979), 338-351.

2. KASHIWADA, T. Some Properties of Locally Conformal Kähler Manifolds, Hokkaido Math. J., 8 (1970), 191-198.

3. KASHIWADA, T. On V-Killing Forms in a Locally Conformal Kähler Manifold with Parallel Lee Form, preprint.

4. KASHIWADA, T. On V-harmonic Forms in compact Locally Conformal Kähler Manifolds with Parallel Lee Form, preprint.

5. YANO, K. Differential Geometry on Complex and Almost Complex Spaces, Pergamon Press, 1965 


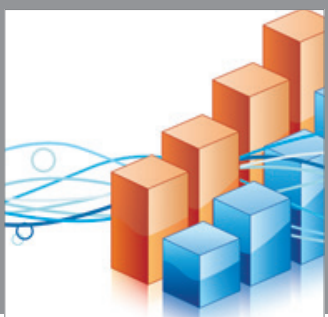

Advances in

Operations Research

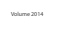

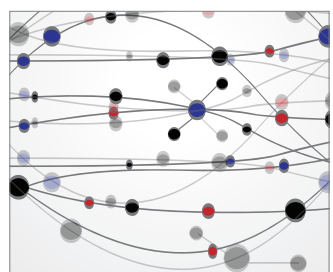

\section{The Scientific} World Journal
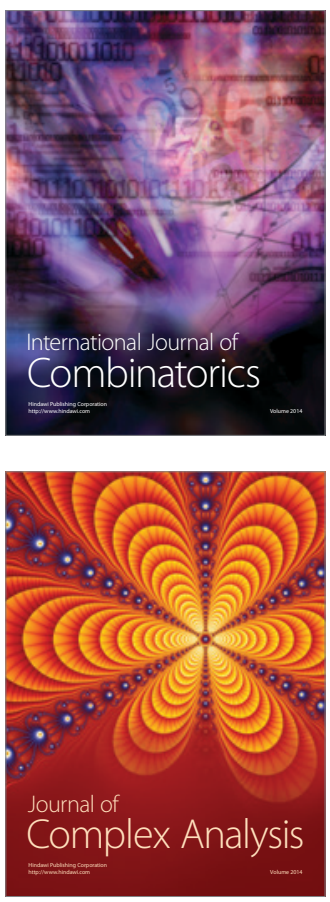

International Journal of

Mathematics and

Mathematical

Sciences
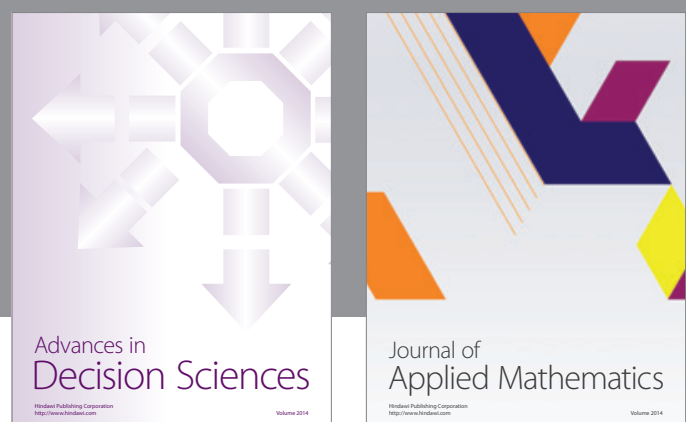

Journal of

Applied Mathematics
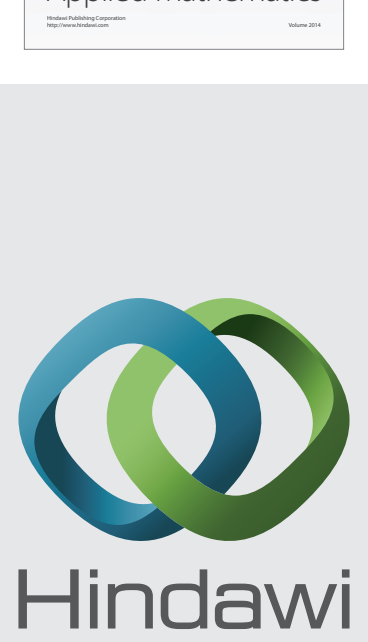

Submit your manuscripts at http://www.hindawi.com
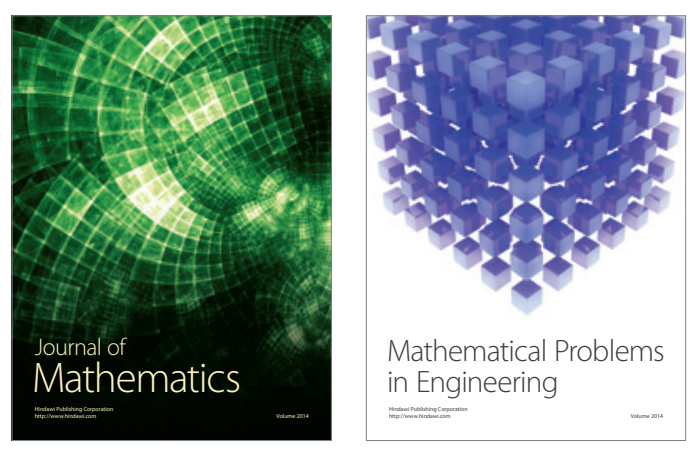

Mathematical Problems in Engineering
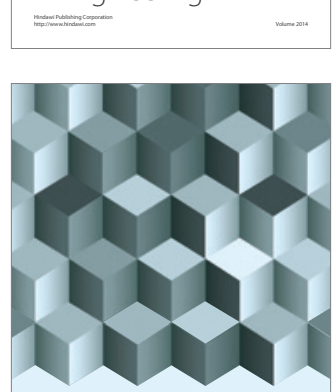

Journal of

Function Spaces
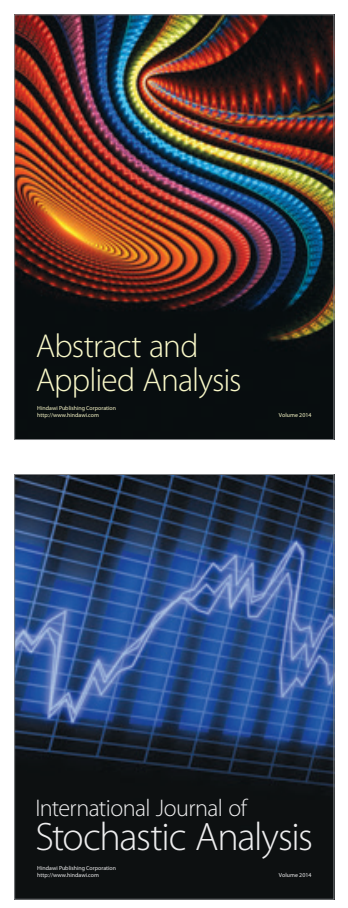

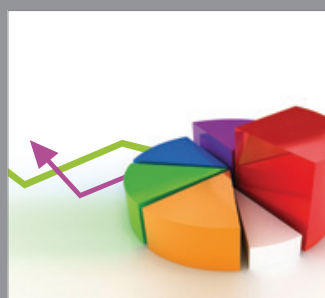

ournal of

Probability and Statistics

Promensencen
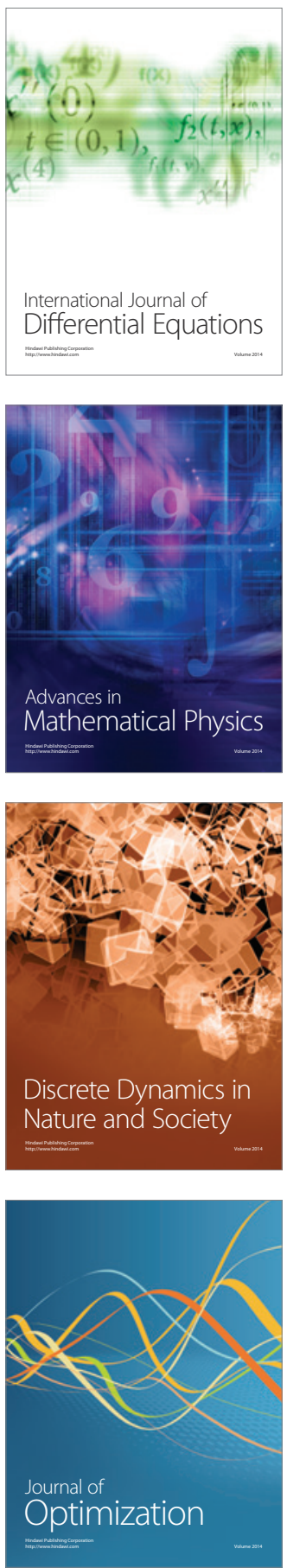